\title{
Corrección de atenuación en equipos PET-RM. Comparación de métodos mediante simulación Monte Carlo
}

\section{Attenuation correction in PET-MRI. A comparison of methods by using Monte Carlo simulation}

\author{
José Pablo Cabello García ${ }^{1}$, Roser Sala-Llonch², Raúl Tudela Fernández ${ }^{3}$, Domènec Ros Puig ${ }^{2,3}$, \\ Javier Pavía Segura ${ }^{1,3}$, Aida Niñerola Baizán ${ }^{1,2,3}$ \\ 1 Servicio de Medicina Nuclear, Hospital Clínic de Barcelona. C. de Villarroel, 170, 08036 Barcelona. \\ 2 Unidad de Biofísica y Bioingeniería, Facultad de Medicina, Universidad de Barcelona. Casanova, 143. 08036 Barcelona. \\ 3 Centro de Investigación Biomédica en Red en Bioingeniería, Biomateriales y Nanomedicina (CIBER-BBN), Barcelona. C \\ Baldiri Reixac, 10-12. 08028 Barcelona.
}

Fecha de Recepción: 15/04/2020 - Fecha de Aceptación: 29/10/2020

La visualización y cuantificación adecuadas de una imagen de tomografía por emisión de positrones (PET) requiere la corrección por la atenuación que sufren los fotones al atravesar el medio. En un equipo híbrido que combina PET con resonancia magnética (RM), la señal de RM no puede convertirse en valores de atenuación de forma directa. En este trabajo se analizaron dos métodos de estimación del mapa de atenuación, el primero basado en segmentación de la imagen RM y el segundo en un promedio de imágenes de tomografía computarizada (TC) a partir de múltiples sujetos. El estudio se realizó utilizando imágenes PET obtenidas mediante simulación Monte Carlo y el parámetro cuantitativo evaluado fue el valor de captación estandarizado relativo (SUVr) tomando como región de referencia el cerebelo.

La comparación de los resultados obtenidos con cada método con los correspondientes al utilizar la imagen TC propia de cada paciente (considerado como gold standard) indica que: 1) ambos métodos pierden exactitud en la zona próxima al tejido óseo, 2) en un análisis de SUVr por regiones, el método que utiliza segmentación a partir de la imagen de RM da mejores resultados con diferencias relativas máximas en torno al $5 \%$ frente al gold standard.

Palabras clave: PET, RM, corrección por atenuación, simulación, Monte Carlo.

Appropriate visualization and quantification in positron emission tomography (PET) imaging requires the correction by the attenuation of photons when crossing the medium. In a hybrid device that combines the PET technique with magnetic resonance imaging (MRI), the signal from MRI cannot be directly converted to attenuation values. In this work, two methods to estimate the attenuation map have been analysed, the first one, based on segmentation from the MRI and the second one, from an average of computed tomography (CT) images from multiple subjects. The study was carried out using PET images obtained by Monte Carlo simulation and the quantitative parameter evaluated was the standardized uptake value ratio (SUVr), taking the cerebellum as reference region.

The results obtained with both methods compared to those obtained using the CT image of each patient (considered as gold standard) show that: 1) the accuracy in the calculation of the total uptake diminishes in the region near the bone tissue, 2) in a SUVr analysis by regions, the method that uses segmentation from the MRI gives better results with maximum relative differences around 5\% compared to the gold standard.

Key words: PET, MRI, attenuation correction, simulation, Monte Carlo.

*Correspondencia: aninerola@ub.edu

https://doi.org/10.37004/sefm/2020.21.2.004 


\section{Introducción}

La corrección por atenuación de fotones es fundamental en la reconstrucción de imágenes de tomografía de emisión de positrones (PET) cerebral tanto para su interpretación visual como para su análisis cuantitativo. Fotones emitidos a diferentes profundidades en el cerebro tienen que atravesar diferentes espesores de tejido para llegar hasta los detectores, provocando una diferencia entre la señal registrada y la real emitida. Para poder realizar dicha corrección es necesario disponer de un mapa de atenuación que almacene los coeficientes de atenuación de los distintos tejidos. Este mapa se puede obtener a partir de una imagen de tomografía computarizada (TC), que aporta información de la densidad electrónica de los tejidos del paciente. Los equipos PET-TC permiten obtener en el mismo equipo la imagen TC y así es posible obtener un mapa de atenuación preciso que coincide espacialmente con la imagen PET del paciente.

En los últimos tiempos están comenzando a instalarse los equipos híbridos que combinan la técnica PET con la imagen de resonancia magnética (RM). Los equipos PET-RM combinan la sensibilidad e información bioquímica de la imagen PET con la precisa información anatómica de la RM gracias al elevado contraste de esta técnica para tejidos blandos.

En consecuencia, la combinación de la técnica PET y la RM puede proporcionar una metodología para explorar el cerebro con información multiparamétrica y complementaria de imágenes. ${ }^{1,2}$ Además, existen equipos PET-RM totalmente integrados basados en fotosensores semiconductores, tales como los fotodiodos de avalancha y los fotomultiplicadores de silicio que permiten la adquisición simultánea de ambos conjuntos de datos de imágenes. Estos equipos poseen varias ventajas frente a la exploración secuencial en equipos PET-TC convencionales, como facilitar la corrección por movimiento o la posibilidad de combinar la imagen PET con estudios funcionales de RM. ${ }^{3-7}$ Aunque también son posibles los estudios multimodales de imágenes PET y RM obtenidos en diferentes equipos, esta simultaneidad permite eliminar los errores de corregistro, que según la patología son difíciles de evitar, así como los debidos a variaciones temporales en el sujeto estudiado. ${ }^{8}$

A pesar de las teóricas ventajas que aportan, tienen un gran inconveniente respecto a los equipos PET-TC. Los equipos PET-RM no disponen de información de la densidad electrónica, debido a que la señal de RM no está directamente relacionada con la atenuación de fotones y, por lo tanto, requieren nuevos métodos para realizar la corrección de atenuación.

En la literatura existente se han propuesto varios métodos para solventar este problema, que se pueden clasificar en tres categorías, ${ }^{9,10}$ así como combinaciones de estas:

1. Aproximaciones basadas en la segmentación de la RM diferenciando entre el hueso y los tejidos blandos seguido de una asignación de coeficientes de atenuación predefinidos para cada tejido. 1,2,9,10

2. Aproximaciones basadas en una imagen pseudo-TC patrón. Esta puede ser derivada de una imagen genérica, ${ }^{11}$ a partir de múltiples imágenes $\mathrm{TC}^{12}$ o generada utilizando métodos de machine learning. ${ }^{3}$

3. Aproximaciones basadas en imágenes de emisión donde el mapa de atenuación es estimado a partir de la imagen de emisión PET, preferiblemente en equipos con time of flight (TOF). ${ }^{5}$ Estos métodos dan resultados comparables a los métodos basados en la aproximación anterior. $1,5,12$

El objetivo de este trabajo es comparar dos métodos basados en los criterios descritos para ver cuál de ellos ofrece mejores resultados. El primero de los métodos está basado en la utilización de un mapa de atenuación obtenido de la imagen de RM del propio paciente y el segundo en la utilización de un mapa obtenido a partir del promedio de TC de una cohorte de sujetos control, comparando ambos con el método de corrección que utiliza la TC propia del paciente, que es considerado el gold standard. ${ }^{4,5}$

El método de la imagen de RM presenta la ventaja de utilizar una imagen del propio paciente adquirida en el mismo equipo. Sin embargo, no se dispone de información real de la atenuación de los fotones a través de los distintos tejidos ni es posible visualizar con la misma precisión que en una imagen TC las estructuras óseas. El método a partir de una TC promedio es fácil de implementar, no necesita ninguna exploración añadida al PET, y utiliza información de densidad electrónica, como en el gold standard. Su gran desventaja es que no tiene información morfológica propia del paciente, ya que el mapa de atenuación está formado por imágenes TC de otros sujetos, aunque se ajuste al sujeto en estudio.

Para la evaluación de los métodos se utilizó la simulación Monte Carlo que permite generar imágenes PET a partir de imágenes de TC y RM de distintos sujetos. La simulación permite crear un marco teórico de referencia y evaluar así el efecto de los distintos métodos sobre imágenes controladas que presentan variabilidad morfológica. 


\section{Material y métodos}

Se simularon estudios PET cerebrales con $\left[{ }^{18} \mathrm{~F}\right]$ $\beta$-Amiloide (A $\beta$ PET) utilizando SimSET $\mathrm{v} 2.9,{ }^{13}$ un código Monte Carlo que permite recrear el recorrido individual de los fotones desde el momento en que se generan en el interior del paciente hasta que llegan al detector (o escapan fuera de este). Para la simulación se partió de las imágenes TC y RM (secuencia T1 adquirida en un escáner Siemens Trio Tim de 3 Tesla) de 11 sujetos para obtener los mapas de actividad y atenuación necesarios para modelar tanto la distribución del trazador como el medio físico que atraviesan los fotones. Posteriormente, los estudios se reconstruyeron utilizando la TC del propio paciente (ATETC) para la corrección de atenuación, y los mapas obtenidos con el método de imagen RM (ATE $E_{R M}$ ) y de TC promedio (ATETCprom).

\section{Mapa de actividad}

El mapa de actividad para la simulación pretende imitar la distribución del trazador que se produce en imágenes PET reales. Para el caso de $\mathrm{A} \beta \mathrm{PET}$, se ha visto que el trazador se distribuye de forma diferente dentro de los diferentes tejidos. En el presente estudio, se simuló una captación homogénea con valores distintos para los principales tejidos cerebrales. Utilizando el software FSL ${ }^{14,15}$ se segmentaron las imágenes de RM de cada paciente en sustancia blanca, sustancia gris, calota y líquido cefalorraquídeo para generar el mapa de actividad de cada sujeto. Para simular una situación de captación normal, los valores utilizados fueron: 4 para el líquido cefalorraquídeo, 20 para la calota, 25 para la sustancia gris y 100 para la sustancia blanca.

\section{Mapa de atenuación}

El proceso para obtener el mapa con los coeficientes de atenuación para cada uno de los métodos fue el siguiente:

- En el método ATETC (gold standard) el mapa de atenuación se creó a partir de la TC del propio paciente. En primer lugar, se corregistró cada imagen de TC con la imagen RM correspondiente usando el software SPM8, ${ }^{16}$ para tener tanto el mapa de atenuación como el de actividad en el espacio de la RM. A continuación, se generó el mapa de contorno de cada sujeto separando el contorno de la cabeza con un umbral de -200 Hounsfield Units $(\mathrm{HU})$, lo que permite conocer donde acaba la cabeza del sujeto y donde comienza el aire que lo rodea. Por último, se identificó qué parte de la cabeza es hueso y qué parte son tejidos blandos, usando un umbral de $500 \mathrm{HU}$. Después de la segmentación se establecieron tres valores diferentes de atenuación para crear el mapa: alta atenuación para tejido óseo $\left(\mu=0.196 \mathrm{~cm}^{-1}\right)$, baja para tejidos blandos $\left(\mu=0.098 \mathrm{~cm}^{-1}\right)$ y nula para aire.

- Para el método ATERM se obtuvo el mapa de atenuación de cada sujeto a partir de la segmentación de la imagen correspondiente de RM. Se segmentó el hueso del cráneo y el contorno de la cabeza usando el programa BET $^{14,15}$ de FSL. De nuevo, como en el caso ATETC, se obtuvo una imagen tridimensional con el perfil de la cabeza del sujeto y otra con el hueso del cráneo, y se asignaron tres valores de coeficientes de atenuación en función de si la región considerada corresponde a tejido blando, tejido óseo o aire.

- En el caso del método ATETcprom el mapa de atenuación se obtuvo a partir de una TC promedio de 13 sujetos diferentes a los del estudio. En primer lugar, se normalizaron las TC de todos los sujetos, usando el software SPM8, para pasarlos al espacio estándar MNI (Montreal Neurological Institute) y reducir las diferencias morfológicas entre ellos. Posteriormente, se obtuvo una imagen promediando las HU de los 13 sujetos vóxel a vóxel. Esta imagen se coregistró con la imagen RM de cada uno de los 11 pacientes del estudio para, una vez en el espacio de cada sujeto, crear los correspondientes mapas de atenuación. Se utilizaron los mismos umbrales de HU que al crear el mapa de atenuación a partir de las imágenes TC propias.

Todas las imágenes utilizadas en este trabajo fueron adquiridas en el centro de diagnóstico por la imagen dentro de los estudios aprobados por el comité de ética del Hospital Clínic de Barcelona, y todos los sujetos dieron su consentimiento informado por escrito.

\section{Simulación}

Se simuló un escáner Siemens Biograph con 32 anillos de detectores de $41.2 \mathrm{~cm}$ de radio basados en germanato de bismuto (BGO), usando ${ }^{18} \mathrm{~F}$ como isótopo. ${ }^{17,18}$ No se modelaron eventos aleatorios ni tiempo muerto en los detectores. Se usó adquisición 3D con span 1 y ventana de energía de 350 a 650 keV. Las dimensiones de los sinogramas generados son 288 píxeles de $2.2 \mathrm{~mm}$ y 288 posiciones angulares. Los 
fotones se separaron en coincidencias verdaderas (trues) y de dispersión (scatter), lo que nos permite una corrección de dispersión ideal. Las simulaciones se realizaron para obtener sinogramas de aproximadamente 80 millones de cuentas. Para la simulación se utilizó el mapa de actividad comentado anteriormente y el mapa de atenuación obtenido con el método ATETC.

\section{Reconstrucción}

La reconstrucción de los sinogramas obtenidos se realizó reproduciendo las condiciones de reconstrucción utilizadas en el equipo Biograph. En primer lugar, se simuló una corrección de dispersión ideal, para lo cual solo se consideraron los sinogramas de las coincidencias verdaderas (trues). Estos sinogramas se corrigieron por atenuación antes de la reconstrucción, usando las herramientas de SimSET, con cada uno de los tres mapas obtenidos con los métodos ATETC, ATE RM y ATE TCprom. A continuación, los sinogramas $3 \mathrm{D}$ ya corregidos se redujeron a un conjunto de sinogramas 2D de un solo corte con un span de 9 mediante el algoritmo SSRB (Single-Slice Rebinning). Posteriormente, se reconstruyeron con un algoritmo basado en subconjuntos ordenados (Ordered Subset Expectation Maximization, OSEM) con 8 subsets y 12 iteraciones utilizando la librería STIR ${ }^{19}$ para los dos últimos procesos. Las dimensiones de las imágenes PET generadas son $128 \times 128 \times 63$ vóxeles y tamaño de vóxel $2.6 \times 2.6 \times 2.4 \mathrm{~mm}^{3}$. El procedimiento global se encuentra resumido en la Fig. 1.

\section{Cuantificación por regiones}

Para comparar los dos métodos ATERM y ATETCprom con el gold standard (ATETC) se analizaron las 3 imágenes PET reconstruidas de cada paciente comparando la captación por regiones.

La captación se cuantificó mediante el parámetro standardized uptake volume ratio (SUVr), ${ }^{20}$ que puede calcularse como el cociente entre la captación en una región y el valor en una región de referencia. Las regiones consideradas fueron las definidas en el atlas Harvard-Oxford ${ }^{21-24}$ cortical. Se empleó este mapa porque segmenta el cerebro en regiones con cierta relevancia en la clínica. ${ }^{25,26}$ El SUVr se calculó para cada región tomando como referencia la captación del cerebelo, y se compararon los resultados de los dos métodos alternativos con el de la TC propia. Para obtener una información más global se realizó el promedio de la variación del SUVr respecto al gold standard en cada región para todos los sujetos. Además, se representaron los resultados obtenidos en mapas cerebrales para tener información visual, tanto de cada de uno de los sujetos como mapas globales para ambos

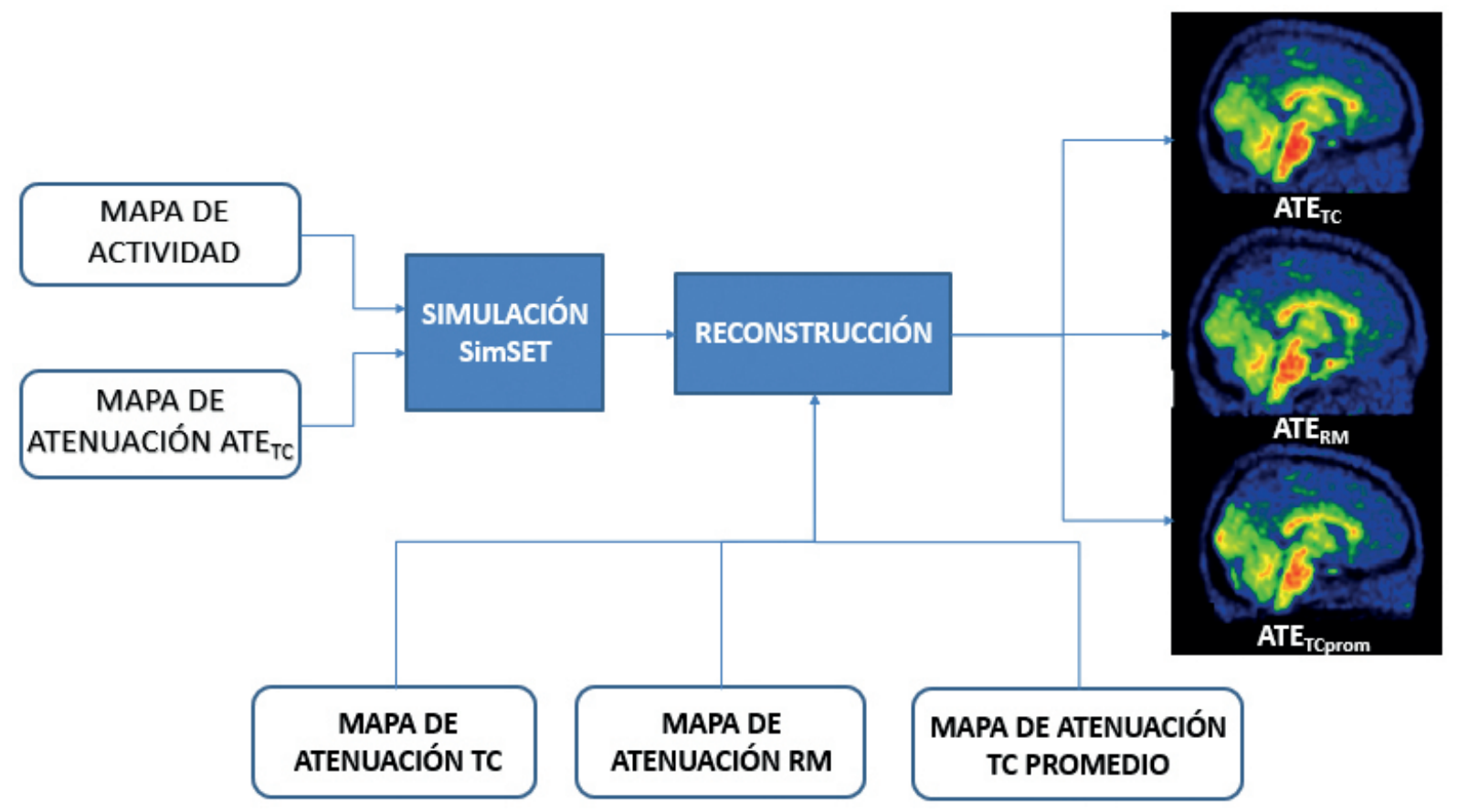

Fig. 1. Proceso de simulación y reconstrucción. Se parte de los mapas de actividad y de atenuación y se reconstruye con cada uno de los 3 mapas de atenuación indicados. 
métodos, $A T E_{R M}$ y ATE TCprom. Los errores relativos (en \%) de SUVr de cada región respecto al gold standard SUVrgs, se calcularon mediante:

$$
\varepsilon_{\mathrm{SUVr}}=\left(\frac{\mathrm{SUVr}}{\mathrm{SUVrgs}}-1\right) \cdot 100
$$

\section{Análisis estadístico}

Para analizar los resultados de la cuantificación por regiones y valorar si las diferencias entre los errores relativos respeto al gold standard encontrados en cada uno de los métodos (ATERM y ATE $E_{T \text { prom }}$ ) son estadísticamente significativas, se utilizó el programa STATA. ${ }^{26}$ El análisis se realizó mediante el test de Fisher Pitman dado que los SUVr relativos de cada uno de los métodos son variables independientes en la muestra de 11 sujetos. Adicionalmente, se han analizado los datos correspondientes a la misma medida del mismo set de sujetos obtenidas con el método gold standard y con cada uno de los métodos de aproximación, utilizando el test Wilcoxon Sign Rank-Sum test. Para considerar un resultado como significativo se empleó una confianza del 95\% ( $p \leq 0.05)$.

\section{Resultados}

La Fig. 2 muestra secciones análogas en los planos sagital y axial de los mapas de atenuación y las correspondientes imágenes reconstruidas con los tres métodos. Se puede observar que visualmente las imágenes PET resultantes son muy similares entre sí.

Para la cuantificación por regiones, se obtuvieron los SUVr para cada uno de los tres métodos y se calculó el $\varepsilon_{\text {SUVr }}$ de los métodos ATERM y ATETCprom respecto al ATETC. Estas diferencias se muestran en dos mapas donde se promedian los resultados de los 11 sujetos (Fig. 3). En estos mapas puede apreciarse que las regiones más internas son las que, en general,

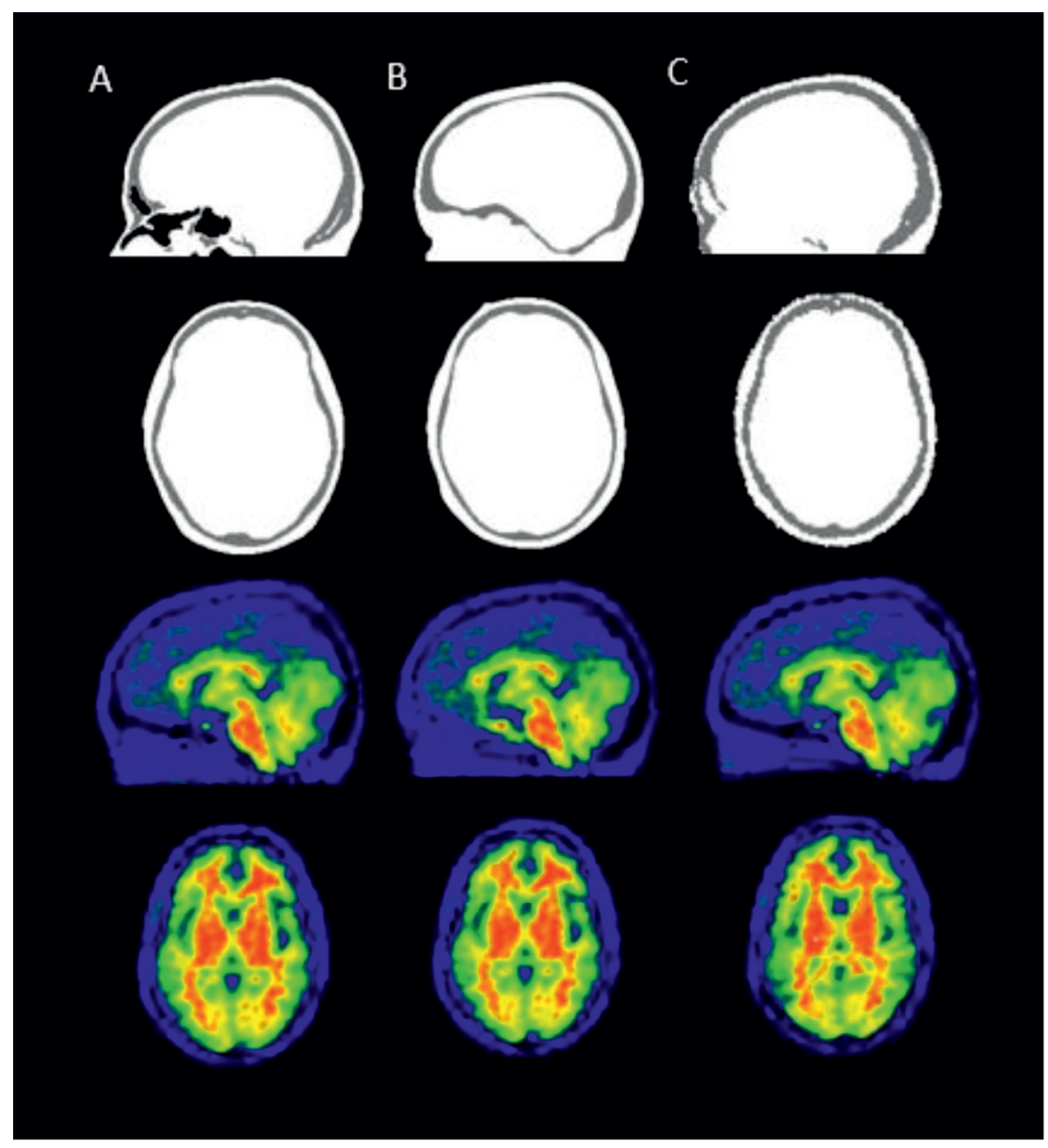

Fig. 2. Secciones sagitales y axiales del mapa de atenuación e imagen PET reconstruida de un mismo sujeto utilizando los métodos $\operatorname{ATE}_{T C}(A), \operatorname{ATE}_{R M}(B)$ y $\operatorname{ATE}_{T C p r o m}(C)$. 


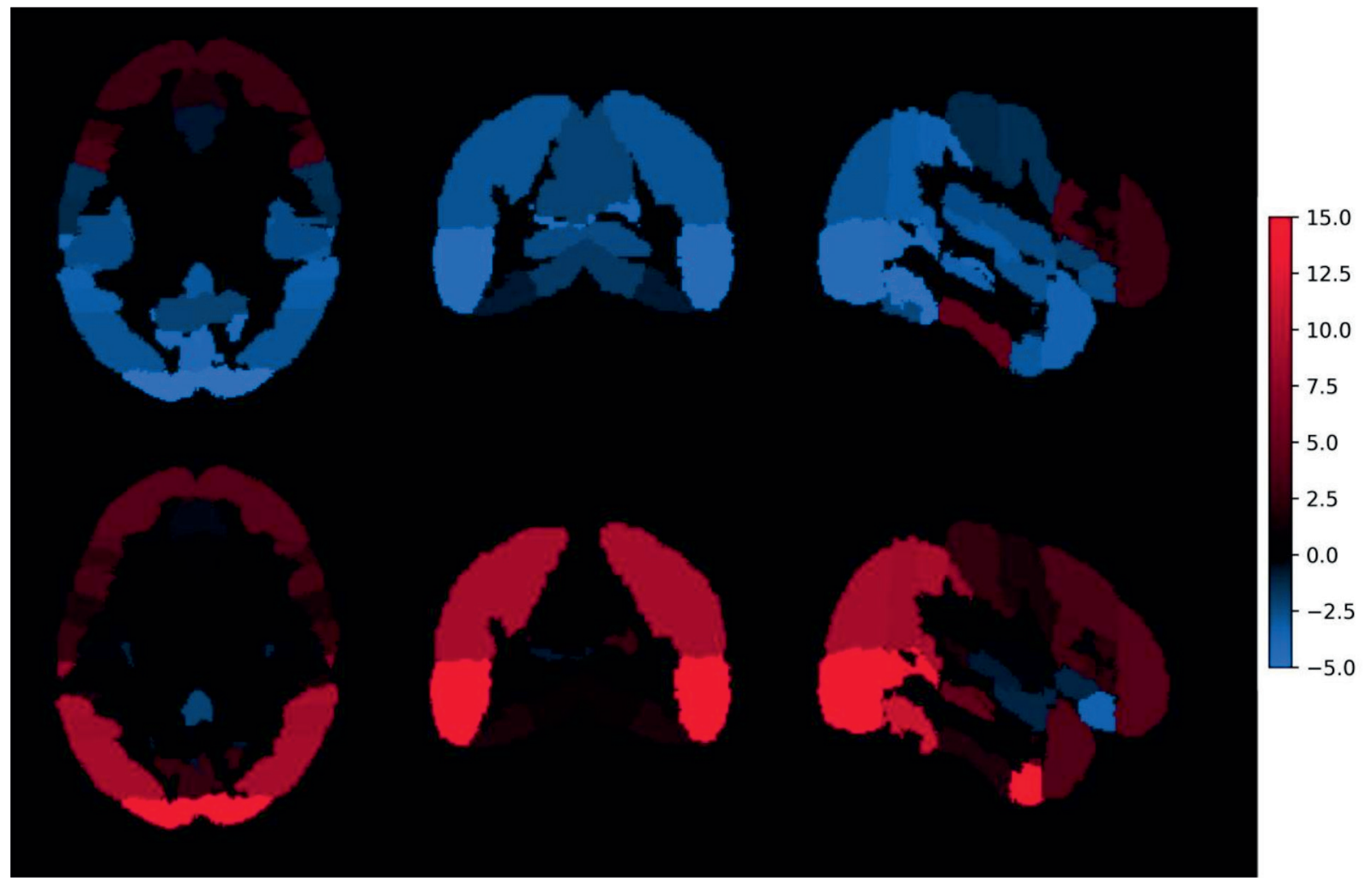

Fig. 3. Secciones axial, coronal y sagital de la imagen de error $\varepsilon_{\text {Suvr }}$ que muestran la diferencia (en \%) entre los mapas de SUVr promedio de los 11 sujetos para cada uno de los métodos empleados respecto al gold standard en las regiones del atlas Harvard-Oxford. La parte superior corresponde al método ATERM y la inferior a ATETCprom.

presentan mayor concordancia con el método ATETC de referencia.

Así mismo y como se ha indicado anteriormente se seleccionaron varias regiones relevantes clínicamente ${ }^{27}$ para comparar los resultados realizando un análisis estadístico más detallado de los errores relativos respecto al gold standard encontrados en cada uno de los métodos propuestos. Las regiones estudiadas fueron: polo frontal, giro frontal medio, giros precentral y postcentral, polo temporal, giro supramarginal, giro angular, corteza lateral occipital, corteza frontal medial, precúneo y cúneo, corteza supracalcarina, polo occipital y tronco cerebral. Se analizó si en dichas regiones existían diferencias estadísticamente significativas entre ambos métodos de reconstrucción. Este análisis se muestra gráficamente en un diagrama de tipo box plot (Fig. 4), para comparar los resultados de forma visual. Hay diferencias significativas entre ambos métodos de reconstrucción, teniendo el método ATE $\mathrm{TCprom} \mathrm{mayor}$ discrepancia respecto al gold standard que marca la TC propia.

En la Tabla 1 se puede observar como en la comparación de muestras apareadas se encontró que la diferencia es estadísticamente significativa $(p<0.05)$ en 10 de las 12 regiones estudiadas para el método a partir de $A_{T} E_{R M}$ y en 8 de las 12 para el método ATETCprom.

\section{Discusión}

Para solucionar el problema de la corrección por atenuación en los equipos PET-RM se han propuesto diversos métodos en la literatura científica, que se pueden dividir en los basados en segmentación de $\mathrm{RM},{ }^{9,10}$ una pseudo-TC patrón ${ }^{12}$ o la imagen de emisión PET. ${ }^{5}$ En este trabajo se usaron los métodos de corrección $A T E_{R M}$, que obtiene el mapa de atenuación a partir de la segmentación de la imagen RM del propio paciente, y ATE $\mathrm{TCprom}_{\mathrm{C}}$, que genera un mapa de atenuación a partir de una imagen TC promedio de múltiples sujetos. Estos dos métodos se analizaron comparándolos con el método de referencia $\mathrm{ATE}_{\mathrm{TC}} .4,5$ Las imágenes PET para el análisis se obtuvieron mediante simulación Monte Carlo de $\left[{ }^{18} \mathrm{~F}\right] \beta$-Amiloide que permite disponer de la distribución real de actividad con la que se genera la imagen, no consume tiempo de tomógrafo y es el único método que tiene la flexibilidad necesaria para plantear un estudio como el que se propone, con distintas distribuciones anatómicas de actividad.

Para comparar los dos métodos ATERM y ATE $_{T C p r o m}$ con el gold standard (ATE $\mathrm{TC}_{\mathrm{T}}$ ) se analizaron las imágenes PET reconstruidas de cada paciente comparando la captación por regiones. De acuerdo con estudios 

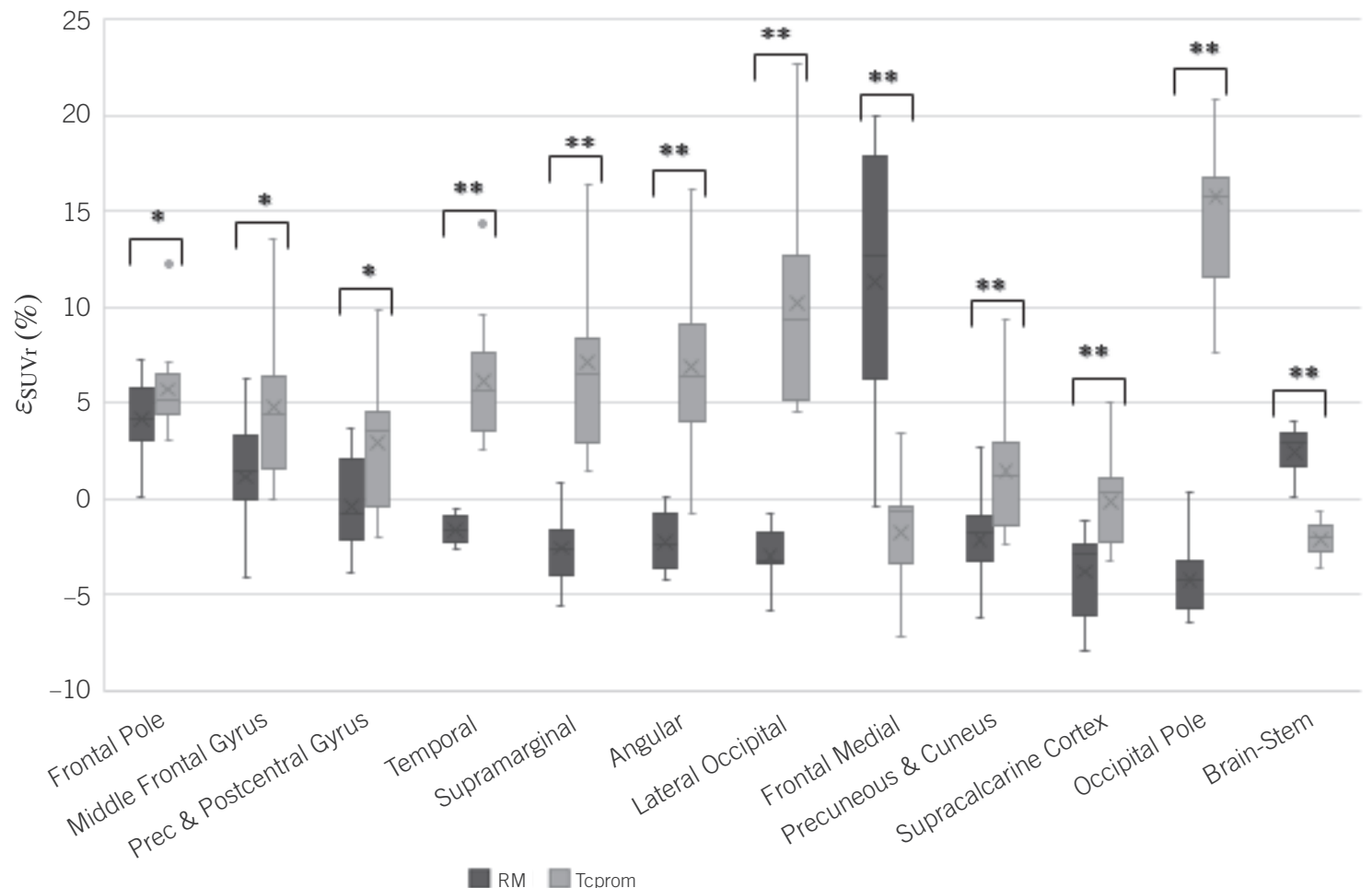

Fig. 4. Diferencia de SUVr promedio entre los métodos empleados y el gold standard en las regiones del atlas Harvard-Oxford. El eje $X$ indica las regiones evaluadas y el eje Y la diferencia porcentual entre el SUVr del método analizado y el gold standard. * indica $p<0.05 \mathrm{y}$ ** indica $p<0.001$ (test Fisher-Pitman).

Tabla 1. SUVr por regiones y diferencias relativas entre los métodos de corrección por atenuación ATERM-ATE $E_{C T}$, ATECTpromATE $_{\text {CT y }}$ ATE $_{\mathrm{RM}}$-ATE $E_{\text {CTprom. }}{ }^{*}$ indica $p<0.05$ (Wilcoxon Sign Rank-Sum test).

\begin{tabular}{|c|c|c|c|c|c|c|c|c|c|}
\hline & \multicolumn{3}{|c|}{ Media SUVr } & \multicolumn{3}{|c|}{ Dif. Relativa } & \multicolumn{3}{|c|}{ Wilcoxon signed rank test } \\
\hline & ATE $_{\text {RM }}$ & ATE $_{\text {TCprom }}$ & ATETC $_{\text {TC }}$ & $\begin{array}{l}\text { ATE }_{\text {RM }}^{-} \\
\text {ATE }_{\text {TC }}\end{array}$ & $\begin{array}{c}\text { ATE }_{\text {TCprom }}{ }^{-} \\
\text {ATE }_{\text {TC }}\end{array}$ & $\begin{array}{c}\text { ATE }_{\mathrm{RM}^{-}} \\
\text {ATE }_{\text {TCprom }}\end{array}$ & $\begin{array}{l}\text { ATE }_{\text {RM }}^{-} \\
\text {ATE }_{\text {TC }}\end{array}$ & $\begin{array}{l}\text { ATE }_{\text {TCprom }}{ }^{-} \\
\text {ATE }^{\text {TC }}\end{array}$ & $\begin{array}{l}\text { ATE }_{\text {RM }^{-}} \\
\text {ATE }_{\text {TCprom }}\end{array}$ \\
\hline Frontal Pole & 0.70 & 0.71 & 0.68 & $4.1 \%$ & $5.7 \%$ & $-1.5 \%$ & $0.001^{*}$ & $0.001^{*}$ & 0.0645 \\
\hline $\begin{array}{l}\text { Middle Frontal } \\
\text { Gyrus }\end{array}$ & 0.83 & 0.86 & 0.82 & $1.3 \%$ & $4.5 \%$ & $-3.1 \%$ & 0.1475 & $0.002^{*}$ & $0.0098 *$ \\
\hline $\begin{array}{c}\text { Precentral \& } \\
\text { Postcentral Gyrus }\end{array}$ & 0.85 & 0.87 & 0.85 & $-0.4 \%$ & $2.4 \%$ & $-2.7 \%$ & 0.6377 & 0.0537 & $0.0059 *$ \\
\hline Temporal & 0.83 & 0.89 & 0.84 & $-1.5 \%$ & $5.4 \%$ & $-6.5 \%$ & $0.001^{*}$ & $0.002^{*}$ & $0.002^{*}$ \\
\hline Supramarginal & 0.80 & 0.87 & 0.82 & $-2.5 \%$ & $5.9 \%$ & $-7.9 \%$ & $0.0049^{*}$ & $0.0068^{*}$ & $0.002^{*}$ \\
\hline Angular & 0.87 & 0.94 & 0.89 & $-2.2 \%$ & $5.8 \%$ & $-7.5 \%$ & $0.002^{*}$ & $0.0049^{*}$ & $0.002^{*}$ \\
\hline Lateral Occipital & 0.85 & 0.96 & 0.88 & $-2.9 \%$ & $9.0 \%$ & $-10.9 \%$ & $0.001^{*}$ & $0.0068^{*}$ & $0.002^{*}$ \\
\hline Frontal Medial & 0.88 & 0.79 & 0.79 & $11.1 \%$ & $0.0 \%$ & $11.1 \%$ & $0.002^{*}$ & 0.2402 & $0.002^{*}$ \\
\hline $\begin{array}{l}\text { Precuneous \& } \\
\text { Cuneus }\end{array}$ & 1.03 & 1.06 & 1.05 & $-2.3 \%$ & $0.8 \%$ & $-3.1 \%$ & $0.0137^{*}$ & 0.5771 & $0.002^{*}$ \\
\hline $\begin{array}{l}\text { Supracalcarine } \\
\text { Cortex }\end{array}$ & 1.13 & 1.16 & 1.17 & $-3.8 \%$ & $-0.6 \%$ & $-3.2 \%$ & $0.001^{*}$ & 0.8311 & $0.0039 *$ \\
\hline Occipital Pole & 0.88 & 1.05 & 0.92 & $-4.2 \%$ & $13.7 \%$ & $-15.8 \%$ & $0.002^{*}$ & $0.002^{*}$ & $0.002^{*}$ \\
\hline Brain-Stem & 1.53 & 1.47 & 1.50 & $2.5 \%$ & $-2.1 \%$ & $4.7 \%$ & $0.001^{*}$ & $0.001^{*}$ & $0.001^{*}$ \\
\hline
\end{tabular}


publicados, ${ }^{28-30}$ los resultados muestran una tendencia del método $A_{T} E_{R M}$ a subestimar la captación del

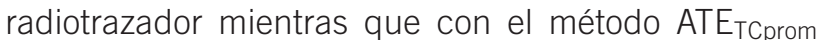
se produce una sobrestimación. Esto puede deberse a que al segmentar las imágenes RM se subestima la cantidad de hueso, mientras que tal como se observa en la Fig. 2, en el método ATETCprom se sobrestima.

Las regiones estudiadas han sido principalmente corticales, debido a su relevancia en la clínica y a su proximidad al hueso, donde se concentran las mayores diferencias relativas, $2,4,12,28$ debido a que la definición del hueso del cráneo provoca las mayores discrepancias en los mapas de atenuación. Los errores relativos de las regiones estudiadas respecto al gold standard se encuentran en torno al 5\% en la mayoría de los casos, con una diferencia relativa menor del $15 \%$ en los casos más desfavorables con el método ATETCprom. Estos resultados son del mismo orden de magnitud que los encontrados en otros estudios que utilizan métodos similares de segmentación de imagen RM y atlas de múltiples sujetos, donde presentan diferencias promedio de entre el $5 \%$ y el 10\%, $3,5,9,12,28,30$ aunque en nuestro estudio únicamente hemos analizado regiones corticales.

En la Fig. 4 se muestran las regiones estudiadas de mayor relevancia y en las que hay diferencias significativas entre los resultados de los métodos utilizados. En casi todas estas regiones se ve menor diferencia relativa con el método $A T E_{R M}$. Existen trabajos previos, como el estudio multicéntrico de Ladefoged et al.. ${ }^{4}$ en donde se hace una comparación entre diversos métodos que muestran que los mejores resultados se obtienen con métodos basados en segmentación a partir de imagen RM, lo que se corresponde con los resultados obtenidos. Si se analizan los datos correspondientes a la misma medida del mismo set de sujetos obtenidas con el método gold standard y con cada uno de los métodos de aproximación, se puede observar en la Tabla 1 la diferencia de resultados entre los métodos y la tendencia a subestimar del ATERM y a sobrestimar

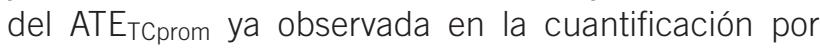
regiones.

Analizando visualmente las imágenes PET resultantes se puede observar que no hay grandes diferencias aparentes entre los dos métodos estudiados y el gold standard, por lo que se puede inferir que ambos métodos permiten generar imágenes aptas para la visualización clínica, pero respecto la cuantificación por regiones, el método $A_{T} E_{R M}$ da mejores resultados.

En todos los resultados se observa una tendencia a que las mayores diferencias se concentren junto al hueso del cráneo. Estas diferencias pueden ser debidas a que la definición del contorno del cráneo es el mayor punto de discrepancia entre los métodos, pero también al efecto de posibles pequeños desa- lineamientos, ${ }^{31}$ pues los corregistros pueden tener pequeños errores y desajustes. Otro posible efecto a considerar es que se utilizó el mismo umbral para definir el hueso en el método ATETCprom que en el método ATE $_{T C}$, sin tener en cuenta que se produce un efecto intrínseco de suavizado de las $\mathrm{HU}$ al promediar las imágenes TC de los diferentes sujetos. Esto puede afectar a la definición del borde del hueso de la cabeza y a su grosor por lo que se considera una limitación del estudio. Es posible que un ajuste diferente de las $\mathrm{HU}$ en este método pueda mejorar ligeramente los resultados obtenidos.

En el caso del método ATERM, se observa una sobrecaptación en las regiones frontales de la base del cráneo. Este es un problema que se ha descrito anteriormente, ${ }^{28,29}$ y es debido a la dificultad de discernir entre cavidades de aire y tejido óseo al segmentar la imagen RM. En nuestro caso, tal como se observa en la Fig. 2, en el mapa de atenuación no aparecen las cavidades de aire de las vías respiratorias, por el contrario, aparece una pequeña zona de hueso que no está en el mapa generado por imagen de TC. Esto es claramente una limitación del método ATE $_{R M}$ para evaluar estas regiones.

\section{Conclusiones}

El método de corrección de atenuación mediante imagen RM del propio paciente y el método mediante TC promedio de múltiples sujetos pierden exactitud en la zona próxima al tejido óseo dando resultados que se desvían menos del $10 \%$ respecto al gold standard en la gran mayoría de las regiones estudiadas.

La mayor limitación encontrada a estos métodos es la concentración de los errores en la zona próxima al cráneo, tanto en la parte anterior como posterior, así como la sobrecaptación en la base del cráneo en el caso del método con imagen RM.

A la vista de estos resultados se puede concluir que podrían utilizarse ambos métodos en función de si se requiere de un análisis cuantitativo, la disponibilidad de tiempo y recursos. Para realizar una cuantificación, los resultados del método con imagen RM son ligeramente mejores para la mayoría de las regiones analizadas.

\section{Bibliografía}

1. Chen $\mathrm{Y}, \mathrm{An} \mathrm{H}$. Attenuation Correction of PET/MR Imaging. Magn Reson Imaging Clin N Am. 2017;25(2):245-55.

2. Izquierdo-Garcia D, Catana C. Magnetic resonance imagingguided attenuation correction of positron emission tomography data in PET/MRI. PET Clin. 2016;11(2):1922-2013. 
3. Liu F, Jang H, Kijowski R, Bradshaw T, McMillan AB. Deep learning MR imaging-based attenuation correction for PET/ MR imaging. Radiology. 2018;286(2):676-84.

4. Ladefoged CN, Law I, Anazodo U, St. Lawrence K, IzquierdoGarcia D, Catana C, et al. A multi-centre evaluation of eleven clinically feasible brain PET/MRI attenuation correction techniques using a large cohort of patients. Neuroimage [Internet]. 2017;147(June 2016):346-59. Available from: http://dx.doi.org/10.1016/j.neuroimage.2016.12.010

5. Cabello J, Lukas M, Rota Kops E, Ribeiro A, Shah NJ, Yakushev I, et al. Comparison between MRI-based attenuation correction methods for brain PET in dementia patients. Eur J Nucl Med Mol Imaging [Internet]. 2016 Nov 20 [cited 2019 Sep 9];43(12):2190-200. Available from: http://www. ncbi.nlm.nih.gov/pubmed/27094314

6. Bai B, Li Q, Leahy RM. MR-Guided PET Image Reconstruction. Semin Nucl Med [Internet]. 2013;43(1):30-44. Available from: http://www.sciencedirect.com/science/article/pii/S000 1299812000827

7. Grimm R, Fürst S, Souvatzoglou M, Forman C, Hutter J, Dregely I, et al. Self-gated MRI motion modeling for respiratory motion compensation in integrated PET/MRI. Med Image Anal [Internet]. 2015;19(1):110-20. Available from: http://dx.doi.org/10.1016/j.media.2014.08.003

8. Datteri RD, Liu Y, D'Haese PF, Dawant BM. Validation of a nonrigid registration error detection algorithm using clinical MRI brain data. IEEE Trans Med Imaging. 2015 Jan 1;34(1):86-96.

9. An HJ, Seo S, Kang H, Choi H, Cheon GJ, Kim HJ, et al. MRI-based attenuation correction for PET/MRI using multiphase level-set method. Journal of Nuclear Medicine. 2016;57:587-93.

10. Okazawa H, Tsujikawa T, Higashino Y, Kikuta K-I, Mori T, Makino A, et al. No significant difference found in PET/MRI CBF values reconstructed with CT-atlas-based and ZTE MR attenuation correction. EJNMMI Res [Internet]. 2019 Mar 19 [cited 2019 Sep 9];9(1):26. Available from: http://www.ncbi. nlm.nih.gov/pubmed/30888559

11. Montandon ML, Zaidi H. Atlas-guided non-uniform attenuation correction in cerebral 3D PET imaging. Neuroimage. 2005;25(1):278-86.

12. Sekine T, Burgos N, Warnock G, Huellner M, Buck A, Ter Voert EEGW, et al. Multi-atlas-based attenuation correction for brain 18F-FDG PET imaging using a time-of-flight PET/MR scanner: Comparison with clinical single-atlas-and CT-based attenuation correction. J Nucl Med. 2016;57(8):1258-64.

13. Haynor DR, Harrison RL, Lewellen TK. The use of importance sampling techniques to improve the efficiency of photon tracking in emission tomography simulations. Med Phys [Internet]. 1991 Sep [cited 2019 Sep 9];18(5):9901001. Available from: http://www.ncbi.nlm.nih.gov/pubmed/1961165

14. Smith SM. Fast robust automated brain extraction. Hum Brain Mapp [Internet]. 2002 Nov [cited 2019 Sep 16];17(3):143-55. Available from: http://www.ncbi.nlm.nih. gov/pubmed/12391568

15. Jenkinson M, Pechaud M, Smith S. BET2: MR-based estimation of brain, skull and scalp surfaces. Elev Annu Meet Organ Hum brain Mapp [Internet]. 2005;17(3): 167. Available from: http://mickaelpechaud.free.fr/these/HBM05.pdf

16. Acton PD, Friston KJ. Statistical parametric mapping in functional neuroimaging: beyond PET and fMRI activation studies. Eur J Nucl Med [Internet]. 1998 Jul [cited 2019 Sep 9];25(7):663-7. Available from: http://www.ncbi.nlm.nih.gov/ pubmed/9741993

17. Marti-Fuster B, Esteban O, Thielemans K, Setoain X, Santos $A$, Ros D, et al. Including anatomical and functional information in MC simulation of PET and SPECT brain studies. Brain-VISET: A voxel-based iterative method. IEEE Trans Med Imaging. 2014;33(10):1931-8.

18. López-González FJ, Moscoso A, Efthimiou N, FernándezFerreiro A, Piñeiro-Fiel M, Archibald SJ, et al. Spill-in counts in the quantification of $18 \mathrm{~F}$-florbetapir on $\mathrm{A} \beta$-negative subjects: the effect of including white matter in the reference region. EJNMMI Phys. 2019;6(1).

19. Thielemans K, Tsoumpas C, Mustafovic S, Beisel T, Aguiar P, Dikaios N, et al. STIR: software for tomographic image reconstruction release 2. Phys Med Biol [Internet]. 2012 Feb 21 [cited 2019 Sep 9];57(4):867-83. Available from: http:// www.ncbi.nlm.nih.gov/pubmed/22290410

20. Jena A, Taneja S, Goel R, Renjen P, Negi P. Reliability of semiquantitative 18 F-FDG PET parameters derived from simultaneous brain PET/MRI: A feasibility study. Eur J Radiol [Internet]. 2014 Jul [cited 2019 Sep 9];83(7):1269-74. Available from: http://www.ncbi.nlm.nih.gov/pubmed/248 13529

21. Makris N, Goldstein JM, Kennedy D, Hodge SM, Caviness VS, Faraone S V., et al. Decreased volume of left and total anterior insular lobule in schizophrenia. Schizophr Res [Internet]. 2006 Apr [cited 2019 Sep 16];83(2-3):155-71. Available from: http://www.ncbi.nlm.nih.gov/pubmed/16448806

22. Frazier JA, Chiu S, Breeze JL, Makris N, Lange N, Kennedy DN, et al. Structural Brain Magnetic Resonance Imaging of Limbic and Thalamic Volumes in Pediatric Bipolar Disorder. Am J Psychiatry [Internet]. 2005 Jul [cited 2019 Sep 16];162(7):1256-65. Available from: http://www.ncbi.nlm. nih.gov/pubmed/15994707

23. Desikan RS, Ségonne F, Fischl B, Quinn BT, Dickerson BC, Blacker D, et al. An automated labeling system for subdividing the human cerebral cortex on MRI scans into gyral based regions of interest. Neuroimage [Internet]. $2006 \mathrm{Jul}$ 1 [cited 2019 Sep 16];31(3):968-80. Available from: http:// www.ncbi.nlm.nih.gov/pubmed/16530430

24. Goldstein JM, Seidman LJ, Makris N, Ahern T, O'Brien LM, Caviness VS, et al. Hypothalamic Abnormalities in Schizophrenia: Sex Effects and Genetic Vulnerability. Biol Psychiatry [Internet]. 2007 Apr 15 [cited 2019 Sep 16];61(8):935-45. Available from: http://www.ncbi.nlm.nih. gov/pubmed/17046727

25. Laforce R, Soucy JP, Sellami L, Dallaire-Théroux C, Brunet F, Bergeron D, et al. Molecular imaging in dementia: Past, present, and future. Vol. 14, Alzheimer's and Dementia. Elsevier Inc.; 2018. p. 1522-52.

26. Boston R, Sumner A. STATA: A Statistical Analysis System for Examining Biomedical Data. Adv Exp Med Biol. 2003 Feb 1;537:353-69.

27. Pagani M, De Carli F, Morbelli S, Öberg J, Chincarini A, Frisoni $\mathrm{GB}$, et al. Volume of interest-based [18F]fluorodeoxyglucose PET discriminates $\mathrm{MCl}$ converting to Alzheimer's disease from healthy controls. A European Alzheimer's Disease Consortium (EADC) study. Neurolmage Clin. 2015;7:34-42.

28. Burgos N, Cardoso MJ, Thielemans K, Modat M, Pedemonte $\mathrm{S}$, Dickson J, et al. Attenuation correction synthesis for 
hybrid PET-MR scanners: Application to brain studies. IEEE Trans Med Imaging. 2014;

29. Catana C, Van Der Kouwe A, Benner T, Michel CJ, Hamm M, Fenchel $\mathrm{M}$, et al. Toward implementing an MRI-based PET attenuation-correction method for neurologic studies on the MR-PET brain prototype. J Nucl Med. 2010.

30. Izquierdo-Garcia D, Hansen AE, Förster S, Benoit D, Schachoff S, Fürst S, et al. An SPM8-based approach for attenuation correction combining segmentation and nonrigid template formation: Application to simultaneous PET/MR Brain Imaging. J Nucl Med. 2014;55(11):1825-30.

31. Martinez-Möller A, Souvatzoglou M, Navab N, Schwaiger M, Nekolla SG. Artifacts from misaligned CT in cardiac perfusion PET/CT studies: Frequency, effects, and potential solutions. J Nucl Med. 2007;48(2):188-93. 
VersaHD.

Powered by

dynamic

Versa $\mathrm{HD}^{\mathrm{m}}$ with Monaco ${ }^{\circledR}$ gives you the clinical

flexibility of high definition dynamic radiosurgery

(HDRS) and conventional RT in a single platform.

HDRS means you can deliver stereotactic treatments

within standard RT time slots, regardless of anatomy

or complexity. And, with advanced image guidance

tools and up to $5 x$ less transmission to non-targeted

regions, you have assurance of end-to-end precision.

Discover how Versa HD can help you meet operational efficiencies while achieving better outcomes for patients.

\section{elekta.com/VersaHD}
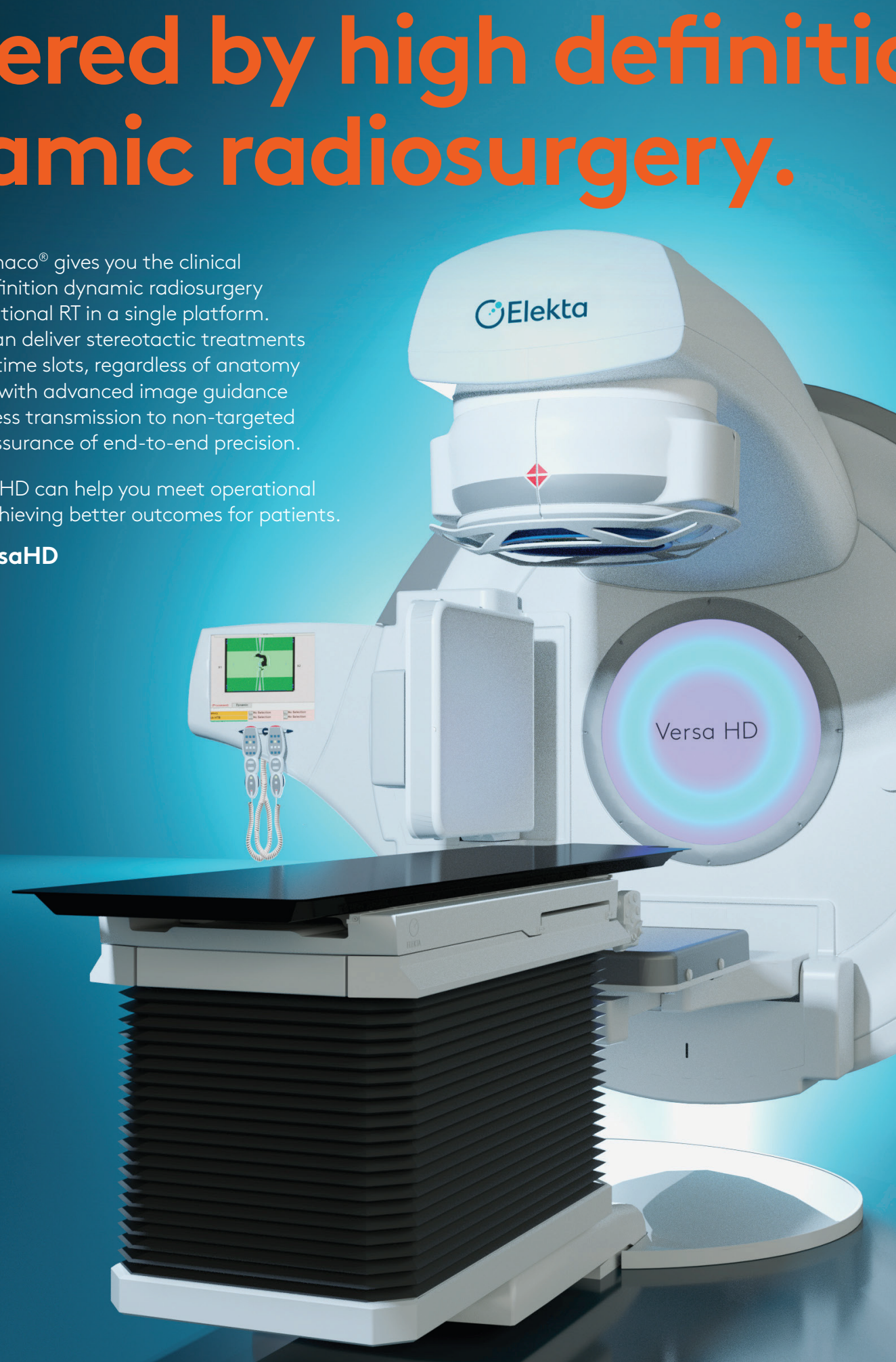